\title{
Effect of natural flavonoids, stilbenes and caffeic acid oligomers on protein glycation
}

\author{
KENROH SASAKI, SYUKO CHIBA and FUMIHIKO YOSHIZAKI \\ Tohoku Pharmaceutical University, Sendai, Miyagi 981-8558, Japan
}

Received May 19, 2014; Accepted June 13, 2014

DOI: 10.3892/br.2014.304

\begin{abstract}
The aim of the present study was to identify the inhibitory activity of natural flavonoids, stilbenes and caffeic acid oligomers on protein glycation. Antioxidant activity was evaluated using 1,1-diphenyl-2-picrylhydrazyl radical-scavenging activity. The production of 3-deoxyglucosone (3-DG) and advanced glycation end products (AGEs) by glycation reactions were determined by high-performance liquid chromatography and fluorescence, respectively. Certain flavonoids, stilbenes and caffeic acid oligomers prevented AGE production and the $\mathrm{IC}_{50}$ values of the compounds were compared. These examined compounds are assumed to suppress AGE generation by inhibiting the increase in 3-DG production through a specific unknown mechanism in the early phase of the Maillard reaction, by inhibiting the generation of active oxygen in the later phase and by suppressing the progression of the reaction. Regular, daily consumption of these compounds in the form of a food or extract is expected to aid in the prevention or inhibition of non-enzymatic amino acid glycation in the living body, which is possibly associated with aging, diabetic complications, arteriosclerotic diseases and Alzheimer's disease, and they may also be effective agents in cosmetics promoting anti-aging.
\end{abstract}

\section{Introduction}

Advanced glycation end products (AGEs) have recently received increasing attention as substances that are directly associated with the generation and development of diabetic complications. The accumulation of AGEs has been reported to be more apparent in the elderly and those with diabetic complications, arteriosclerotic diseases and Alzheimer's disease $(1,2)$. Numerous studies have revealed the association of AGEs with

Correspondence to: Dr Kenroh Sasaki, Tohoku Pharmaceutical University, 4-1 Komatsushima 4-chome, Aoba-ku, Sendai, Miyagi 981-8558, Japan

E-mail: kenrs@tohoku-pharm.ac.jp

Key words: 1,1-diphenyl-2-picrylhydrazyl radical-scavensing activity, 3-deoxyglucosone, advanced glycation end products production, flavonoid, stilbene, caffeic acid oligomer neurodegenerative diseases (3-5), the growth and metastasis of malignant tumors (6) and inflammatory reactions. In the process of generating AGEs, Amadori rearrangement-induced compounds generate 3-deoxyglucosone (3-DG) (7). 3-DG is considered to be a significant reaction intermediate since its generation is associated with oxidative stress, it is cytotoxic (8) to cancer and normal cells, and it demonstrates mutagenic properties.

Natural products, such as crude drugs, reportedly include organic compounds with significant antioxidant properties. Among them, flavonoid compounds from Rutaceae, such as the Citrus genus, exhibit biological activity in various fields and their antioxidant properties are well known in the prevention of chronic disease progression (9). Daily consumption of flavonoids has also been reported to reduce the risk of chronic diseases, including cardiovascular diseases and cancer. In particular, polymethoxyflavone, which is a component of citrus fruits (10), has been reported to reduce inflammation (11) and have an antiproliferative effect on human cancer cells (12).

Rosmarinic acid, which is a phenylpropanoid derivative, is generally present in Boraginaceae and Lamiaceae and exhibits a variety of biological activities, including significant anti-inflammatory and antioxidant effects. With regards to the mechanisms of the anti-inflammatory effect, inhibition of 5-lipoxygenase (13), inhibition of histamine release from mast cells and the scavenging of superoxide radicals (also known as the antioxidant effect) (14) have been reported previously.

The present study, based on the aforementioned, aim to examine the inhibitory effects of flavonoids, stilbenes and caffeic acid polymers, which are known as natural antioxidant substances, on non-enzymatic amino acid glycation.

\section{Materials and methods}

Materials. Nobiletin (15), piceatannol (16) were purified from a peel of Citrus unshiu and Callistemon rigidus, respectively. Rosmarinic acid and lithospermic acid (17) were purified from Lithospermum erythrorhizon. Bovine serum albumin (BSA) and aminoguanidine (Sigma-Aldrich, St. Louis, MO, USA); baicalin, quercetin and naringin (Nacalai Tesque, Inc., Kyoto, Japan); and baicalein, kaempferol, hesperidin and hesperetin (Wako Pure Chemical Industries, Ltd., Osaka, Japan) were purchased. Lithospermic acid B and epirabdosiin were provided by Dr Tabata (Hokkaido Mitsui Chemicals, Inc., Hokkaido, Japan). 
Measurement of 1,1-diphenyl-2-picrylhydrazyl (DPPH) radical-scavenging activity. A modified version of a previous study (18) was used to measure the free radical-scavenging activity of the pure compounds with DPPH. In a 96-well microplate, $100 \mu 1$ sample at different concentrations (in absolute $\mathrm{MeOH}$ ) was added into wells containing $100 \mu \mathrm{l}$ $0.06 \mathrm{mM}$ DPPH in $\mathrm{MeOH}$ and mixed well. The absorbance was measured at $510 \mathrm{~nm}$ exactly by a microplate reader (ImmunoMini NJ-2300; Biotech Co., Ltd., Tokyo, Japan) after $30 \mathrm{~min}$ and the percentage inhibition was calculated. $\mathrm{IC}_{50}$ values express the sample concentration required to scavenge $50 \%$ of the DPPH-free radicals. All the samples were assessed in triplicate.

AGE production inhibitory assay. The assay was carried out in accordance with a previous study (19). D-Glucose (10\%) and BSA (1\%) were dissolved in phosphate-buffered saline (PBS) (pH 7.2). The samples were dissolved in PBS with 5\% DMSO. The glucose-BSA solution $(900 \mu 1)$ and sample solution $(100 \mu \mathrm{l})$ were mixed under asepsis and the mixture was incubated for 22 weeks at $37^{\circ} \mathrm{C}$ with $100 \%$ humidity. The assay samples were collected at $0,1,3,5,9$ and 22 weeks. Fluorescence (F) was measured at $440 \mathrm{~nm}$, excited at $375 \mathrm{~nm}$, following the dilution of the mixture (1:10). The inhibitory activity was calculated as follows: Inhibitory activity $(\%)=$ [1 - (F sample - F sample Blank $_{1}$ ) / (F control - F normal)] x 100. For the control (F control), the PBS buffer was used instead of the sample solution in PBS. The F sample constituted the sample solution and glucose-BSA solution; $\mathrm{F}$ sample Blank $_{\text {was }}$ the sample solution without incubation; and $\mathrm{F}$ normal was the glucose-BSA solution without incubation. The detection of 3-DG was performed in accordance with a previous study (20). Briefly, following the sample collection, 3-DG was labeled by 2,3 -diaminonaphthalene for $14 \mathrm{~h}$ at $4^{\circ} \mathrm{C}$, using $0.05 \%$ 2,3-butanedione as an internal standard. The reaction mixture was analyzed by high-performance liquid chromatography (HPLC) [column, COSMOSIL 5PE-MS, 4.6x250 mm (Nacalai Tesque, Inc.); mobile phase, $50 \mathrm{mM}$ phosphorus acid- $\mathrm{CH}_{3} \mathrm{CN}-\mathrm{MeOH}$ (60:20:20), $1.0 \mathrm{ml} / \mathrm{min}$; detector, UV at $268 \mathrm{~nm}]$ at $20^{\circ} \mathrm{C}$.

Statistics. $\mathrm{IC}_{50}$ values were expressed as the mean of triplicate experiments in DPPH-radical scavenging activity. The fluorescence values of advanced glycation-end products were expressed as the mean of triplicate experiments. The values of 3 -DG formation were expressed as the mean \pm standard error of the mean. Significance was tested by assessing the effects of the compounds compared to aminoguanidine, and $\mathrm{P}<0.05$ was considered to indicate a statistically significant difference.

\section{Results}

Effects of flavonoids and caffeic acid polymers on DPPH radical-scavenging activity. The inhibitory effects of eight types of flavonoid, two types of stilbene and five types of caffeic acid polymer were examined on the DPPH radical-scavenging actvity. The results are shown in Table I. The $\mathrm{IC}_{50}$ of quercetin was the highest for the flavonoids, at $53.75 \mu \mathrm{M}$. Stilbenes showed stronger antioxidant effects than flavonoids; the $\mathrm{IC}_{50}$ values of piceatannol and resveratrol
Table I.DPPH-radical scavenging activity of plant-polyphenols ${ }^{\mathrm{a}}$.

\begin{tabular}{lr}
\hline Polyphenols & $\mathrm{IC}_{50}, \mu \mathrm{M}$ \\
\hline Baicalin & 60.06 \\
Baicalein & 72.96 \\
Nobiletin & $>100$ \\
Kaempferol & 173.18 \\
Quercetin & 53.75 \\
Naringin & $>100$ \\
Hesperidin & $>100$ \\
Hesperetin & $>100$ \\
Piceatannol & 46.80 \\
Resveratrol & 47.16 \\
Caffeic acid (CA) & 93.78 \\
Rosmarinic acid (RA) & 43.68 \\
Lithospermic acid (LA) & 34.26 \\
Lithospermic acid B (LAB) & 10.26 \\
Epirabdosiin (RAB) & 12.38 \\
\hline
\end{tabular}

${ }^{\text {aD }}$ ata are shown as mean (n=6). DPPH, 1,1-diphenyl-2-picrylhydrazyl.

were 46.8 and $47.16 \mu \mathrm{M}$, respectively. Among the caffeic acid polymers, lithospermic acid B exhibited the strongest inhibitory effect, followed by epirabdosiin, lithospermic acid, rosmarinic acid and caffeic acid. In particular, lithospermic acid $\mathrm{B}$ showed the strongest radical-scavenging effect, with an $\mathrm{IC}_{50}$ of $10.26 \mu \mathrm{M}$. The $\mathrm{IC}_{50}$ values of epirabdosiin and lithospermic acid were 12.38 and $34.26 \mu \mathrm{M}$, respectively, suggesting that the radical-scavenging effects are enhanced depending on the degree of polymerization.

Effects of flavonoids and caffeic acid polymers on the generation of 3-DG. Sugar-dicarbonyls, such as 3-DG, are useful markers for the effects of protein glycation inhibitors and defense mechanisms against protein glycation in the body as they show high-level reactivity. A sample was collected 20 weeks after initiating the incubation and 3-DG was derivatized in the sample with 2,3-diaminoaphthalene to examine the effect of the flavonoids, stilbenes and caffeic acid polymers using HPLC. 2,3-Butanedione was used as an internal standard. The amount of 3-DG generated by BSA $(50 \mathrm{mg} / \mathrm{ml})$ and glucose $(500 \mu \mathrm{M})$ was suppressed by the addition of these compounds as compared to the control. Among the flavonoids, kaempferol and naringin showed significant differences compared to aminoguanidine (Fig. 1). Among the stilbenes, resveratrol and piceatannol exhibited suppressing effects that were as strong as aminoguanidine (Fig. 2). Among the caffeic acid polymers, lithospermic acid, lithospermic acid B and epirabdosiin showed significant differences, whereas caffeic acid and rosmarinic acid did not. The amount of 3-DG generated with lithospermic acid, lithospermic acid B and epirabdosiin was $35.84,38.89$ and $36.2 \mu \mathrm{g} / \mathrm{ml}$, respectively, compared to $83.34 \mu \mathrm{g} / \mathrm{ml}$ with the control (Fig. 3).

Effects of the flavonoids and caffeic acid polymers on the generation of AGE-derived fluorescent materials. The 


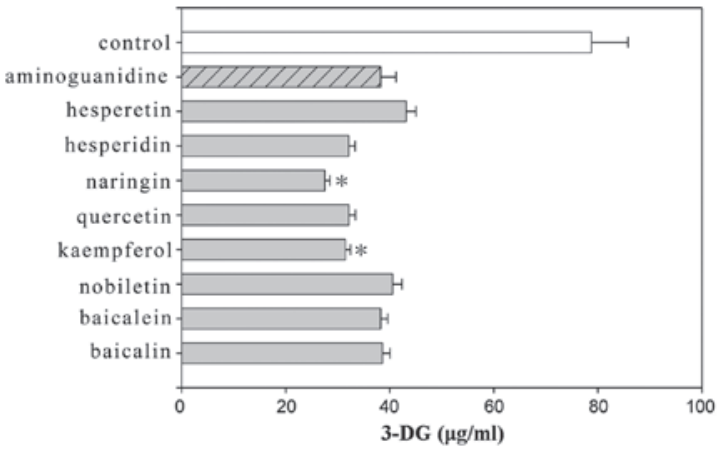

Figure 1. Effects of flavonoids on the formation of 3-deoxyglucosone (3-DG). Data are shown as mean \pm standard error of the mean $(n=6)$. "P<0.05, significantly different from the aminoguanidine value.

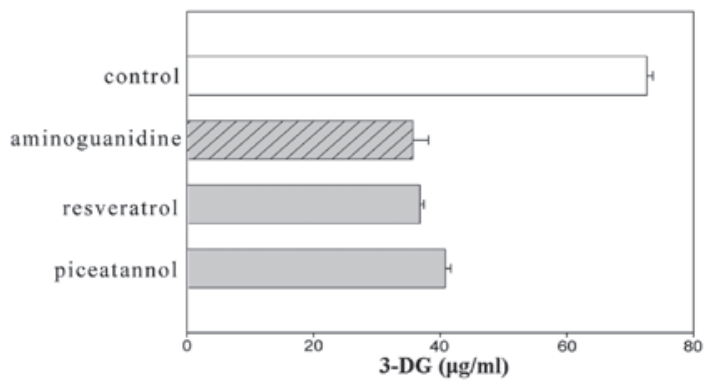

Figure 2. Effects of stilbenes on the formation of 3-deoxyglucosone (3-DG) Data are shown as mean \pm standard error of the mean $(n=6)$.

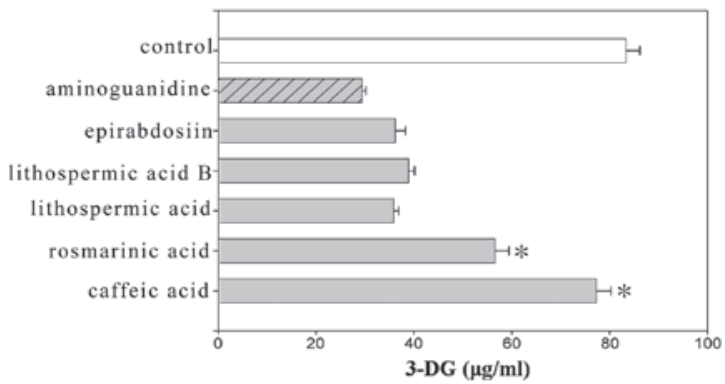

Figure 3. Effects of caffeic acid derivatives on the formation of 3-deoxyglucosone (3-DG). Data are shown as mean \pm standard error of the mean $(n=6)$. ${ }^{*} \mathrm{P}<0.05$, significantly different from the aminoguanidine value.

increase of fluorescence attributed to AGE-derived fluorescent materials was examined with fluorescence spectrophotometry $1,3,4$ and 9 weeks after the initiation of the incubation. In all the flavonoids, stilbenes and caffeic acid polymers compounds, the intensity of the fluorescence decreased compared to the control (Figs. 4-6). Aminoguanidine, which was a positive control, inhibited the intensity of AGE-derived fluorescence by $\sim 30 \% 9$ weeks after the initiation of the incubation. The amino groups of aminoguanidine bind to the keto group of an Amadori product (ketoamine) in the early phase of protein glycation and the keto group of 3-DG in the late phase, and the subsequent reactions are considered to inhibit the progression (21). Baicalin, baicalein and nobiletin, which are flavonoids, inhibited the intensity of AGE-derived fluorescence by $31.4,34.8$ and $32.4 \%$, respectively. For the stilbenes, piceatannol showed $27.8 \%$ inhibition, which was similar to
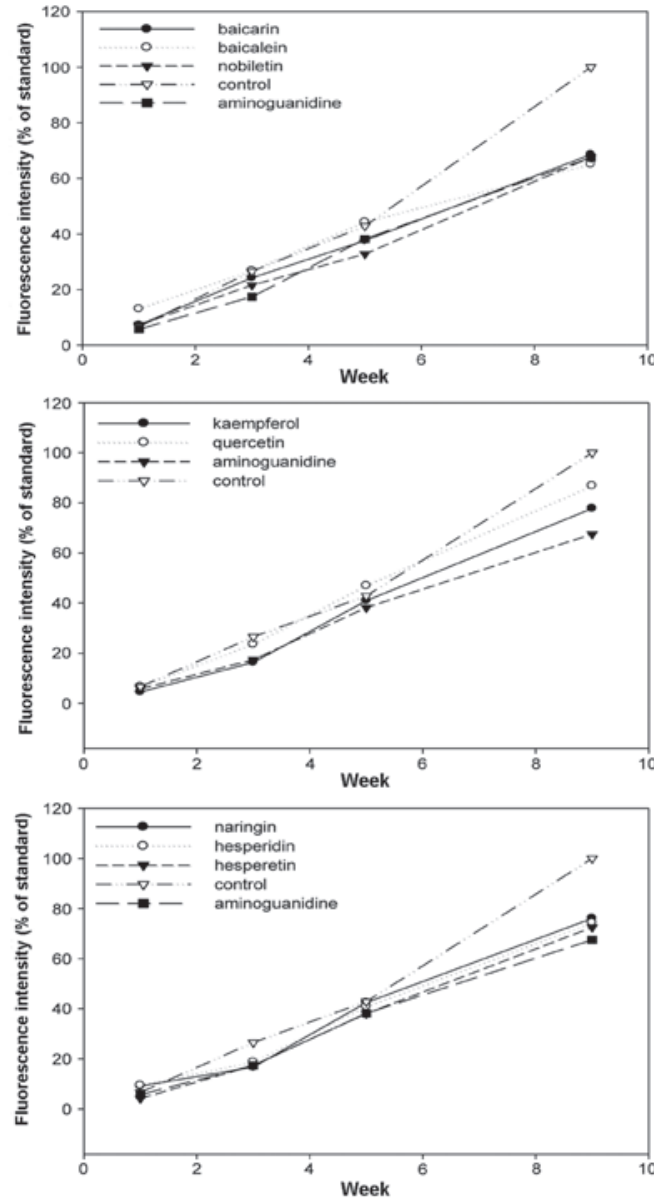

Figure 4. Inhibitory effects of flavonoids against the formation of advanced glycation-end products. Data are shown as mean $(n=6)$.

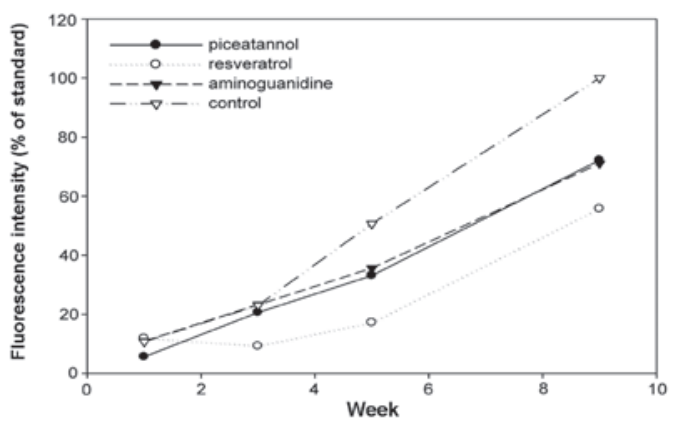

Figure 5. Inhibitory effects of stilbenes against the formation of advanced glycation-end products. Data are shown as mean $(n=6)$.

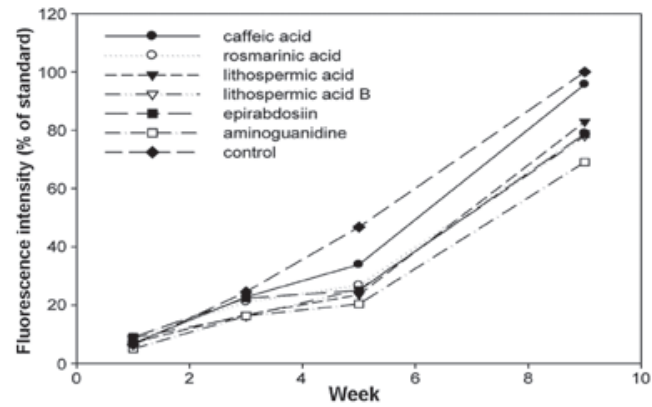

Figure 6. Inhibitory effects of caffeic acid derivatives against the formation of advanced glycation-end products. Data are shown as mean $(n=6)$. 
the inhibition exhibited by aminoguanidine, and resveratrol showed $44.2 \%$ inhibition, which was stronger compared to aminoguanidine. The inhibitory effects of the caffeic acid polymers were low compared to those of the flavonoids and stilbenes, at $20 \%$.

\section{Discussion}

Flavonoids, stilbenes and caffeic acid polymers all have antioxidant properties, and it is suggested that they inhibit the generation of AGEs by inhibiting oxidative stress reactions during the Maillard reaction and by suppressing the progression of these reactions in the later phases. Oxidation is not involved in the formation of Amadori rearrangement compounds, whereas oxidation is associated with the generation of AGEs, including pentosidine (22) and carboxymethyllysine (23), and fluorescent as well as bridged structures, which are certain AGE characteristics (24). As the Amadori compounds are converted to AGEs by active oxygen, they become sources of active oxygen. Highly-reactive intermediates, including 3-DG, rapidly promote the generation of AGEs and AGEs that fluoresce by generating 3-DG, such as pentosidine and pyrropyridine, increase as the reaction progresses. The majority of the plant polyphenols used in the present study strongly suppressed 3-DG generation and, as a result, the generation of AGEs. However, AGEs are assumed to be generated through the reaction pathway for the auto-oxidation of glucose, which is not associated with 3-DG. In this pathway, highly-reactive dicarbonyl compounds, including glyoxal and methylglyoxal, react with proteins and generate AGEs (25). Therefore, a clear positive correlation could not be shown between the antioxidant property and inhibition of AGE generation. These examined compounds are assumed to suppress AGE generation by inhibiting the increase of $3-\mathrm{DG}$ through a specific unknown mechanism in the early phase of the Maillard reaction, by inhibiting the generation of active oxygen in the later phase and by suppressing the progression of the reaction. Further examinations are required to determine whether each compound affects both the early and late phases, or only one of them.

Flavonoids are oxidized by a direct reaction with free radicals, the free radicals are stabilized and the active oxygen species are directly eliminated. The possibility that these plant polyphenols directly bind to proteins (26), which are substrates that would inhibit the bonding of sugars and proteins and suppress the promotion of the Maillard reaction, was also suggested. Caffeic acid polymers have a tannic effect. The interactions between tannins and proteins are associated with hydrogen bonding and hydrophobic interactions based on van der Waals forces, and the repelling of water and polymerization generally intensifies the tannic effect (27). The involvement of the carboxyl group and its steric structure, as well as the degree of polymerization, have been suggested to have important roles in inhibiting the Maillard reaction.

These compounds are generally consumed more as multiple components rather than individually. Regular, daily consumption of these compounds in the form of a food or extract is expected to aid in the prevention or inhibition of non-enzymatic amino acid glycation in the living body, which is possibly associated with aging, diabetic complications, arteriosclerotic diseases and Alzheimer's disease, and they may also be effective agents in cosmetics promoting anti-aging.

\section{References}

1. Barlovic DP, Soro-Paavonen A and Jandeleit-Dahm KA: RAGE biology, atherosclerosis and diabetes. Clin Sci (Lond) 121: 43-55, 2011.

2. Vitek MP, Bhattacharya K, Glendening JM, Stopa E, Vlassara H, Bucala R, Manogue K and Cerami A: Advanced glycation end products contribute to amyloidosis in Alzheimer disease. Proc Natl Acad Sci USA 91: 4766-4770, 1994.

3. Sasaki N, Toki S, Chowei H, Saito T, Nakano N, Hayashi Y, Takeuchi $\mathrm{M}$ and Makita Z: Immunohistochemical distribution of the receptor for advanced glycation end products in neurons and astrocytes in Alzheimer's disease. Brain Res 888: 256-262, 2001.

4. Sasaki N, Takeuchi M, Chowei H, Kikuchi S, Hayashi Y, Nakano N, Ikeda H, Yamagishi S, Kitamoto T, Saito T and Makita Z: Advanced glycation end products (AGE) and their receptor (RAGE) in the brain of patients with Creutzfeldt-Jakob disease with prion plaques. Neurosci Lett 326: 117-120, 2002.

5. Kikuchi S, Shinpo K, Ogata A, Tsuji S, Takeuchi M, Makita Z and Tashiro K: Detection of N epsilon-(carboxymethyl)lysine (CML) and non-CML advanced glycation end-products in the anterior horn of amyotrophic lateral sclerosis spinal cord. Amytroph Lateral Scler Other Motor Neuron Disord 3: 63-68, 2002.

6. Abe R, Shimizu T, Sugawara H, Watanabe H, Nakamura H, Choei H, Sasaki N, Yamagishi S, Takeuchi M and Shimizu H: Regulation of human melanoma growth and metastasis by AGE-AGE receptor interactions. J Invest Dermatol 122: 461-467, 2004.

7. Dyer DG, Blackledge JA, Thorpe SR and Baynes JW: Formation of pentosidine during nonenzymatic browning of proteins by glucose. Identification of glucose and other carbohydrates as possible precursors of pentosidine in vivo. J Biol Chem 266: 11654-11660, 1991.

8. Kato H, van Chyuyen N, Shinoda T, Sekiya F and Hayase F: Metabolism of 3-deoxyglucosone, an intermediate compound in the Maillard reaction, administered orally or intravenously to rats. Biochem Biophys Acta 1035: 71-76, 1990.

9. Rice-Evans C, Spencer JP, Schroeter H and Rechner AR: Bioavailability of flavonoids and potential bioactive forms in vivo. Drug Metabol Drug Interact 17: 291-310, 2000.

10. Kang GJ, Han SC, Yi EJ, Kang HK and Yoo ES: The inhibitory effect of premature Citrus unshiu extract on atopic dermatitis in vitro and in vivo. Toxicol Res 27: 173-180, 2011

11. Baek SH, Kim SM, Nam D, Lee JH, Ahn KS, Choi SH, Kim SH, Shim BS, Chang IM and Ahn KS: Antimetastatic effect of nobiletin through the down-regulation of $\mathrm{CXC}$ chemokine receptor type 4 and matrix metallopeptidase-9. Pharm Biol 50: 1210-1218, 2012

12. Manthey JA and Guthrie N: Antiproliferative activities of citrus flavonoids against six human cancer cell lines. J Agric Food Chem 50: 5837-5843, 2002.

13. Yamamoto H, Sakakibara J, Nagatsu A and Sekiya K: Inhibitors of arachidonate lipoxygenase from defatted perilla seed. J Agric Food Chem 46: 862-865, 1998.

14. Nakamura Y, Ohto Y, Murakami A and Ohigashi H: Inhibitory effects of curcumin and tetrahydrocurcuminoids on the tumor promoter-induced reactive oxygen species generation in leukocytes in vitro and in vivo. Jpn J Cancer Res 89: 361-370, 1998.

15. Sasaki K and Yoshizaki F: Nobiletin as a tyrosinase inhibitor from the peel of Citrus fruit. Biol Pharm Bull 25: 806-808, 2002.

16. Kobayashi K, Ishihara T, Khono E, Miyase T and Yoshizaki F: Constituents of stem bark of Callistemon rigidus showing inhibitory effects on mouse alpha-amylase activity. Biol Pharm Bull 29: 1275-1277, 2006.

17. Murata T, Sasaki K, Sato K, Yoshizaki F, Yamada H, Mutoh H, Umehara K, Miyase T, Warashina T, Aoshima H, et al: Matrix metalloproteinase-2 inhibitors from Clinopodium chinense var. parviflorum. J Nat Prod 72: 1379-1384, 2009.

18. Lee SK, Mbwambo ZH, Chung H, Luyengi L, Gamez EJ Mehta RG, Kinghorn AD and Pezzuto JM: Evaluation of the antioxidant potential of natural products. Comb Chem High Throughput Screen 1: 35-46, 1998. 
19. Valencia JV, Weldon SC, Quinn D, Kiers GH, DeGroot J, TeKoppele JM and Hughes TE: Advanced glycation end product ligands for the receptor for advanced glycation end products: biochemical characterization and formation kinetics. Anal Biochem 324: 68-78, 2004.

20. Yamada H, Miyata S, Igaki N, Yatabe H, Miyauchi Y, Ohara T, Sakai M, Shoda H, Oimomi $M$ and Kasuga M: Increase in 3-deoxyglucosone levels in diabetic rat plasma. Specific in vivo determination of intermediate in advanced Maillard reaction. J Biol Chem 269: 20275-20280, 1994.

21. Edelstein D and Brownlee M: Mechanistic studies of advanced glycosylation end product inhibition by aminoguanidine. Diabetes 41: 26-29, 1992.

22. Sady C, Khosrof S and Nagaraj R: Advanced Maillard reaction and crosslinking of corneal collagen in diabetes. Biochem Biophys Res Commun 214: 793-797, 1995.

23. Ahmed MU, Thorpe SR and Baynes JW: Identification of $\mathrm{N}$ epsilon-carboxymethyllysine as a degradation product of fructoselysine in glycated protein. J Biol Chem 261: 4889-4894, 1986.
24. Reddy S, Bichler J, Wells-Knecht KJ, Thorpe SR and Baynes JW: $\mathrm{N}$ epsilon-(carboxymethyl)lysine is a dominant advanced glycation end product (AGE) antigen in tissue proteins. Biochemistry 34: 10872-10878, 1995.

25. Thornalley PJ, Langborg A and Minhas HS: Formation of glyoxal, methylglyoxal and 3-deoxyglucosone in the glycation of proteins by glucose. Biochem J 344: 109-116, 1999.

26. Wei J, Jin F, Wu Q, Jiang Y, Gao D and Liu H: Molecular interaction study of flavonoid derivative $3 \mathrm{~d}$ with human serum albumin using multispectroscopic and molecular modeling approach. Talanta 126: 116-121, 2014.

27. Richard T, Lefeuvre D, Descendit A, Quideau S and Monti JP: Recognition characters in peptide-polyphenol complex formation. Biochim Biophys Acta 1760: 951-958, 2006. 